\title{
Ácido tranexâmico e hemostasia em cirurgia de revascularização do miocárdio com circulação extracorpórea
}

\author{
Guilherme F. VARGAS*, João Nelson R. BRANCO*, Ana Hercilia S. GUIMARĀES*, Cecília KOBATA*, \\ Espedito T. V. F. SILVA*, Carlos Alberto TELES*, Carlos Arnulfo A. LA ROTTA*, M. L. A. BATISTA
} FILHO*, José Carlos S. ANDRADE*, Ênio BUFFOLO*

VARGAS, G. F.; BRANCO, J. N. R.; GUIMARÃES, A. H. S.; KOBATA, C.; SILVA, E. T. V. F.; TELES, C. A.; LA ROTTA, C. A. A.; BATISTA FILHO, M. L. A.; ANDRADE, J. C. S.; BUFFOLO, E. - Ácido tranexâmico e hemostasia em cirurgia de revascularizaçāo do miocárdio com circulaçāo extracorpórea. Rev. Bras. Cir. Cardiovasc., 7(4):275-282, 1992.

RESUMO: $O$ antifibrinolítico sintético ácido tranexâmico (Transamin (®) foi avaliado em seus efeitos hemostáticos e poupadores de transfusōes homólogas, em pacientes submetidos a revascularização do miocárdio com circulação extracorpórea (CEC). Quarenta pacientes receberam placebo e 55 pacientes foram operados sob o efeito do ácido tranexâmico na dose de $10 \mathrm{~g}$ endovenosa no trans-operatório $(2 \mathrm{~g}$ administrados na induçāo anestésica e os restantes $8 \mathrm{~g}$ nas 4 horas seguintes de cirurgia, de modo contínuo). 0 ácido tranexâmico, na dosagem utilizada, demonstrou possuir efeito hemostático impressionante, promovendo uma reduçāo no débito pelos drenos torácicos da ordem de $47 \%$ nas 12 horas de P.O., $42,5 \%$ nas 24 horas de $P$. O.e $40,5 \%$ até a retirada dos drenos, em relaçāo ao grupo-controle $(p<0,05)$. 0 ácido tranexâmico promoveu uma menor utilizaçāo de concentrado de glóbulos homólogos por paciente, porém, diferença estatisticamente significante foi demonstrada apenas nas 24 horas de P.O. com 1,025 unidades/paciente no grupo controle e 0,333 unidades/paciente no grupo-estudo. Com relaçāo às complicaçōes pós-operatórias, houve maior número de alterações neurológicas sem seqüelas $(2,5 \%$ contra $12,7 \%)$ e alteraçōes de creatinina $(5 \%$ contra $10,9 \%)$ no grupo com o ácido tranexâmico. Tais alteraçōes foram atribuídas à alta dosagem da droga. Como conclusāo, não se recomenda o uso rotineiro do ácido tranexâmico em pacientes submetidos a revascularizaçāo do miocárdio na dosagem de $10 \mathrm{~g}$ endovenoso no trans-operatório, mas, devido ao evidente efeito hemostático da droga, aconselha-se maiores investigaçōes a respeito da dosagem e modo de administraçāo ideais.

DESCRITORES: ácido tranexâmico, cirurgia cardíaca; hemostasia cirúrgica; miocárdio, revascularização, cirurgia; circulaçāo extracorpórea.

\section{INTRODUÇĀO}

O sangramento cirúrgico intra e pós-operatório sempre foi assunto de preocupação e estudo para os cirurgiōes cardiovasculares, por ser ocorrência freqüente, algumas vezes grave, e usualmente mais presente que em outras especialidades cirúrgicas. Tal sangramento muitas vezes é devido a fator vascular localizado, porém, freqüentemente, é conseqüência das alteraçōes de coagulaçāo induzidas pelo processo da circulação extracorpórea: hepari- nização sistêmica e a agressão sangüínea dos circuitos de CEC ${ }^{30,31}$.

Tais alteraçōes do sistema homeostático são desencadeadas pela exposição do sangue a superfícies não fisiológicas, não endoteliais, e cuja responsabilidade com relação à determinaçāo do sangramento é discutível, segundo os autores.

Assim, alguns autores $1,15,17,25,47$ referem alteração plaquetárias quantitativas e qualitativas após

Trabalho realizado no Serviço de Cirurgia Cardiovascular da Escola Paulista de Medicina. Sảo Paulo, SP, Brasil

Apresentado ao $19^{\circ}$ Congresso Nacional de Cirurgia Cardíaca. Sảo Paulo. SP, 7 a 9 de maio de 1992.

* Do Serviço de Cirurgia Cardiovascular da Escola Paulista de Medicina.

Endereço para separatas: Rua Borges Lagoa $783,5^{\circ}$ andar. 04038 São Paulo, SP, Brasil 
VARGAS, G. F.; BRANCO, J. N. R.; GUIMARĀES, A. H. S.; KOBATA, C.; SILVA, E. T. V. F.; TELES, C. A.; LA ROTTA, C. A. A.; BATISTA FILHO, M. L. A.; ANDRADE, J. C. S.; BUFFOLO, E. - Ácido tranexâmico e hemostasia em cirurgia de revascularização do miocárdio com circulaçāo extracorpórea. Rev. Bras. Cir. Cardiovasc., 7(4):275-282, 1992.

a CEC. Outros $5,8,18,35$ referem ativaçăo do fator XII de contato (Hageman) durante a CEC, apesar da heparinização sistêmica adequada, com conseqüente desencadeamento da cascata e consumo dos fatores de coagulaçāo. Outros referem como principal causa de sangramentos após a CEC a ativaçāo do sistema fibrinolítico 12, 21, 32, 33, 48 .

As alteraçōes de coagulaçăo aparentemente eram mais evidentes e graves nos primórdios da CEC, tendo melhorado com o desenvolvimento de modernos circuitos e oxigenadores, porém ainda estāo presentes, contribuindo para o sangramento cirúrgico. Tal sangramento sem dúvida é um determinante da necessidade de transfusōes sangüíneas, as quais merecem muita atenção por parte do cirurgiāo, pelos seus riscos potenciais e possibilidade de transmissão de doenças, temendo-se hoje em dia a AIDS, já que são discretos portadores soro negativos que podem levar 5 anos ou mais para se tornarem soro positivos 6 .

Métodos para se evitar as transfusöes de sangue homólogo sảo descritos na literatura, como autotransfusāo de sangue preservado ou criopreservado, aparelho Cell-Saver na cirurgia ou autotransfusăo de sangue drenado no pós-operatório ${ }^{*}$. Entre tais métodos existe a possibilidade do uso de drogas ditas hemostáticas, assunto ainda controverso na literatura ${ }^{9}$.

Drogas citadas na literatura com efeitos hemostáticos em CEC incluem o acetato de desmopressina ${ }^{44}$, a prostaciclina ${ }^{16} \mathrm{e}$, merecendo destaque, as drogas anfibrinolíticas.

Os antifibrinolíticos existentes e disponiveis comercialmente são três: a aprotinina (Trasylol ®), proteína de peso molecular de aproximadamente 6.500 daltons, conhecida desde 1930 como inibidor enzimático pancreático, atualmente extraída do pulmāo de bovinos ${ }^{6} ; 0$ ácido épsilon-aminocapróico, aminoácido sintético, descrito por S. OKAMOTO em 1953 e o ácido tranexâmico, também um aminoácido sintético, descrito pelo mesmo autor anterior em 1964 38, 39. 40 .

Existe controvérsia na literatura sobre os efeitos hemostáticos e o uso dos antifibrinolíticos em CEC, com alguns autores referindo efeitos hemostáticos positivos $29,33,35,48-51$ e outros nāo ${ }^{19,20,41}$, contra-indicando o uso dos mesmos em CEC, por possível ocorrência de efeitos colaterais.

Destacam-se na literatura, nos últimos anos, vários trabalhos europeus referindo excelentes efeitos hemostáticos e poupadores de transfusões homólogas, com o uso do antibrinolítico natural (aprotinina), administrado em altas dosagens 6, 7, 13, 14, 22 . 23, 37.

O presente trabalho visa documentar novos achados no que diz respeito a efeitos hemostáticos, poupadores de transfusōes homólogas e colaterais do uso de uma alta dosagem do antifibrinolítico sintético ácido tranexâmico, em pacientes coronariopatas submetidos a revascularizaçāo do miocárdio com CEC, segundo um estudo duplo-cego.

\section{CASUÍSTICA E MÉTODOS}

De janeiro a agosto de 1992, os pacientes adultos por nós submetidos a cirurgia cardiovascular foram aleatoriamente divididos em um grupo tratado com ácido tranexâmico e um grupo-controle. A grande maioria dos pacientes era coronariopata, e em menor número valvapata, portadores de aneurismas de aorta e portadores de anomalias congênitas. Para homogeneizar os grupos com relação ao tipo de operaçăo, que sem dúvida é um fator de sangramento pós-operatório, propusemo-nos estudar os coronariopatas, excluindo as reoperaçōes, as revascularizaçōes sem CEC e revascularizaçōes com procedimentos associados (aneurisma de VE ou troca valvar concomitante).

Desta maneira obtivemos um grupo controle de 40 pacientes e um grupo estudo de 55 pacientes. Tratou-se de trabalho "duplo-cego", pois o pessoal médico nāo sabia a que grupo cada paciente pertencia, cabendo a identificaçäo dos mesmos e o preparo da droga ou placebo ao pessoal da enfermagem.

O protocolo da administração do ácido tranexâmico foi de $2 \mathrm{~g}$ durante a induçāo anestésica, em 20 minutos, e $8 \mathrm{~g}$ em gotejamento contínuo, com bomba de infusāo, durante as $4-5$ horas do ato operatório. O grupo controle recebeu igual volume de placebo (S. G. $5 \% 250 \mathrm{ml}$ ).

Os pacientes foram operados com a mesma técnica cirúrgica, que inclui heparinização sistêmica inicial de 400 UI de heparina/kg, hipotermia sistêmica de $28^{\circ} \mathrm{C}$ e proteçāo miocárdica com cardioplegia sangüínea. O controle da heparinizaçāo durante a CEC foi realizado pelo TCA, assim como a neutralizaçāo da heparina por sulfato de protamina, de acordo com técnica convencional.

As médias dos dados gerais, exames laboratoriais, uso de drogas que podem influir no sangramento e dados cirúrgicos dos dois grupos estāo referidos na Tabela 1.

Foram avaliados e comparados os seguintes dados nos dois grupos:

- Sangramento pelos drenos torácicos na $1^{\mathrm{a}}, 2^{\mathrm{a}}$, $3^{a}, 4^{a}, 6^{a}, 12^{a}$ e $24^{a}$ horas de P.O., de maneira cumulativa, assim como o sangramento total até a retirada dos drenos, em $\mathrm{ml} / \mathrm{m}^{2}$. 
VARGAS, G. F.; BRANCO, J. N. R.; GUIMARÄES, A. H. S.; KOBATA, C.; SILVA, E. T. V. F.; TELES, C. A.; LA ROTTA, C. A. A.; BATISTA FILHO, M. L. A.; ANDRADE, J. C. S.; BUFFOLO, E. - Ácido tranexâmico e hemostasia em cirurgia de revascularizaçāo do miocárdio com circulação extracorpórea. Rev. Bras. Cir. Cardiovasc., 7(4):275-282, 1992.

TABELA 1

FATORES QUE PODEM INFLUIR NO SANGRAMENTO DADOS CIRÚRGICOS

\begin{tabular}{lcc}
\hline & CONTROLE & ESTUDO \\
\hline Idade (anos) & 58,5 & 62,5 \\
Sexo feminino & $20 \%$ & $21,8 \%$ \\
Área de Superfície Corpórea (m) & 1,84 \\
Uso de Heparina no Pré-operatório & 1,83 & $31,3 \%$ \\
Uso Recente de Estreptoquinase (3 dias) & $33,3 \%$ & $3,9 \%$ \\
Uso Recente de Aspirina (14 dias) & $0 \%$ & $19,6 \%$ \\
Creatinina no Pré-operatório & $12,8 \%$ & 1,02 \\
Hematócrito no Pré-operatório & 1,05 & $38,96 \%$ \\
Hematócrito de Chegada à UTI & $38,46 \%$ & $35,39 \%$ \\
Tempo de CEC (minutos) & $33,82 \%$ & 97,41 \\
No de Pontes & 94,26 & 3,4 \\
Uso da Artéria Torácica Interna E & 3,5 & $96,07 \%$ \\
Heparina Total Administrada (mg) & $89,74 \%$ & 496,92 \\
Protamina Total Administrada (mg) & 492,95 & 740,49 \\
TCA Após Protamina (segundos) & 736,33 & 122 \\
Oxigenador de Bolhas & $120 "$ & $56,86 \%$ \\
Oxigenador de Membranas & $58,97 \%$ & $43,13 \%$ \\
Hemodiluição Total na CEC & $41,02 \%$ & $49,01 \%$ \\
\hline
\end{tabular}

- Sangramento em $\mathrm{ml} / \mathrm{m}^{2}$ por hora de P.O. (entre a $4^{\mathrm{a}}$ e $6^{\mathrm{a}}$ hora, $6^{\mathrm{a}}$ e $12^{\mathrm{a}}$ hora e $12^{\mathrm{a}}$ e $24^{\mathrm{a}}$ hora; o sangramento por hora foi estimado dividindose o sangramento nestes períodos respectivamente por 2, 6 e 12).

- Quantidade de sangue homólogo reposto em unidades de concentrado de glóbulos, no intraoperatório, nas 24 h de P.O., após 24 h de P.O. e total da internação.

- Comparadas a incidência das seguintes complicaçōes pós-operatórias: IAM per-operatório; complicaçōes neurológicas sem aqüelas (desorientaçāo têmporo-espacial, agitaçāo psicomotora ou sonolência); complicaçōes neurológicas com seqüelas; complicaçōes renais (níveis de creatinina sérica em dobro em relação ao préoperatório); intubação prolongada ( 24 h ou mais) e incidência de reoperaçāo por sangramento.

\section{Método Estatístico}

Para a análise dos resultados, foram utilizados testes não paramétricos, em decorrência da natureza das distribuiçōes das variáveis em estudo. As comparaçōes entre os dois grupos de pacientes foram feitas através do teste de Mann-Whitney, para duas amostras independentes (SIEGEL) ${ }^{45}$. De acordo com o tamanho da amostra, utilizou-se a estatística $U$ ou $Z$ do teste de Mann-Whitney, com aproximaçāo à curva normal.

Com o objetivo de estudar as associaçōes entre variáveis (presença ou ausência) entre grupos, utilizou-se o teste do Qui-Quadrado ou, levando-se em conta as restriçōes de COCHRAN ${ }^{10}$, o teste de Fischer para tabelas $2 \times 2^{45}$.
Na comparaçāo de danos de um mesmo grupo em momentos distintos (sangramento por hora), recorreu-se à Análise de variância por postos de Friedman e teste de comparaçōes múltiplas (HOLLANDER \& WOLFE ${ }^{24}$ )

Em todos os testes fixou-se em 0,05 ou $5 \%$ (alfa menor ou igual a 0,05 ) o nivel para rejeiçāo da hipótese de nulidade.

\section{RESULTADOS}

A análise estatística dos dados mostrados na Tabela 1 não revelou diferenças significantes entre grupos com relaçāo aos fatores que podem influir no sangramento pós-operatório e na necessidade

GRÁFICO

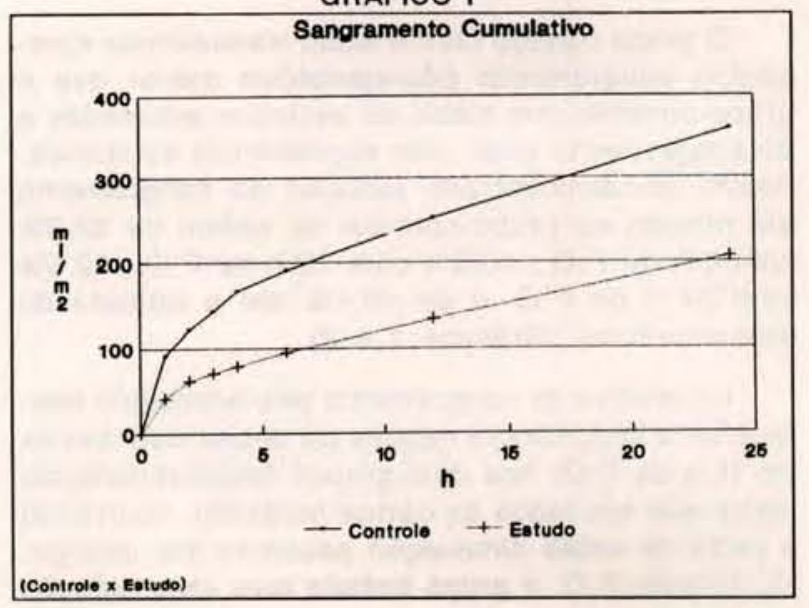


VARGAS, G. F.; BRANCO, J. N. R.; GUIMARĀES, A. H. S.; KOBATA, C.; SILVA, E. T. V. F.; TELES, C. A.; LA ROTTA, C. A. A.; BATISTA FILHO, M. L. A.; ANDRADE, J. C. S.; BUFFOLO, E. - Ácido tranexâmico e hemostasia em cirurgia de revascularizaçāo do miocárdio com circulaçāo extracorpórea. Rev. Bras. Cir. Cardiovasc., 7(4):275-282, 1992.

\section{GRÁFICO 2}

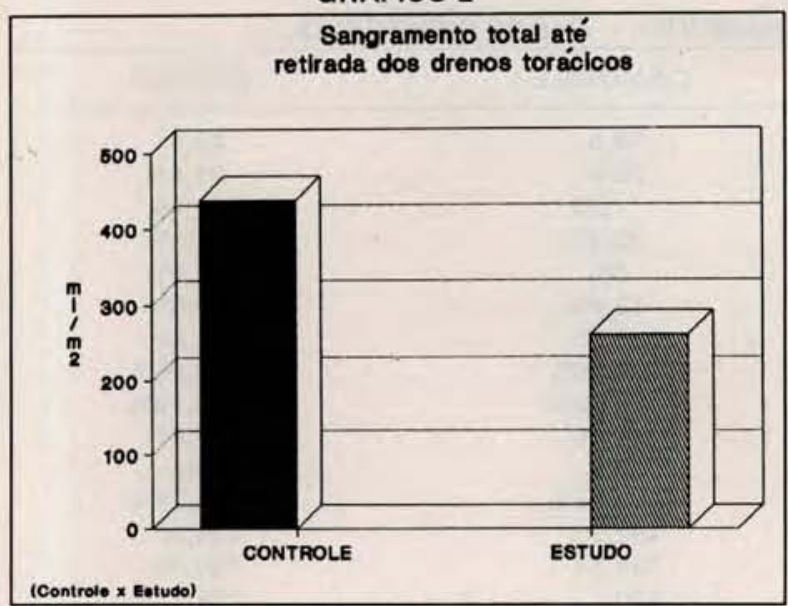

GRÁFICO 3

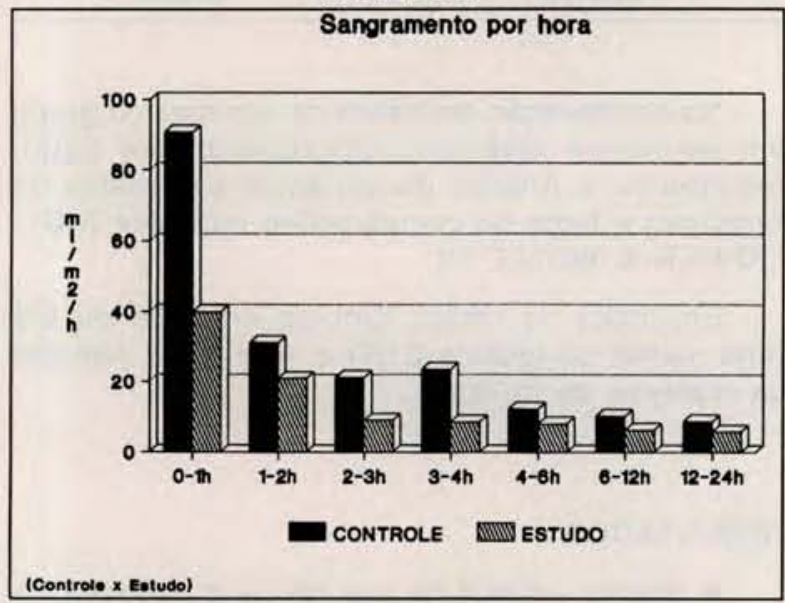

de reposiçāo de sangue homólogo, porém houve diferença significante com relaçăo à idade (média de 58,5 anos para o grupo-controle e 62,5 anos para o grupo-estudo).

O grupo tratado com o ácido tranexâmico apresentou sangramento pós-operatório menor que o grupo-controle, em todos os períodos estudados e no sangramento total, com significância estatística. Assim, foi demonstrado reduçāo do sangramento em relaçāo ao grupo-controle da ordem de $52,2 \%$ com 6 h de P.O.; $46,8 \%$ com 12 h de P.O.; $42,5 \%$ com 24 h de P.O. e de $40,4 \%$ até a retirada do dreno torácico (Gráficos 1 e 2).

$\mathrm{Na}$ análise do sangramento pós-operatório destaca-se a importância relativa do débito dos drenos na $1^{\mathrm{a}} \mathrm{h}$ de P.O. nos dois grupos (estatisticamente maior que em todos os outros horários), ocorrendo a partir de entāo diminuiçāo paulativa dos débitos. $\mathrm{Na} 1^{\mathrm{a}} \mathrm{h}$ de P.O. o grupo tratado teve uma reduçäo
GRÁFICO 4

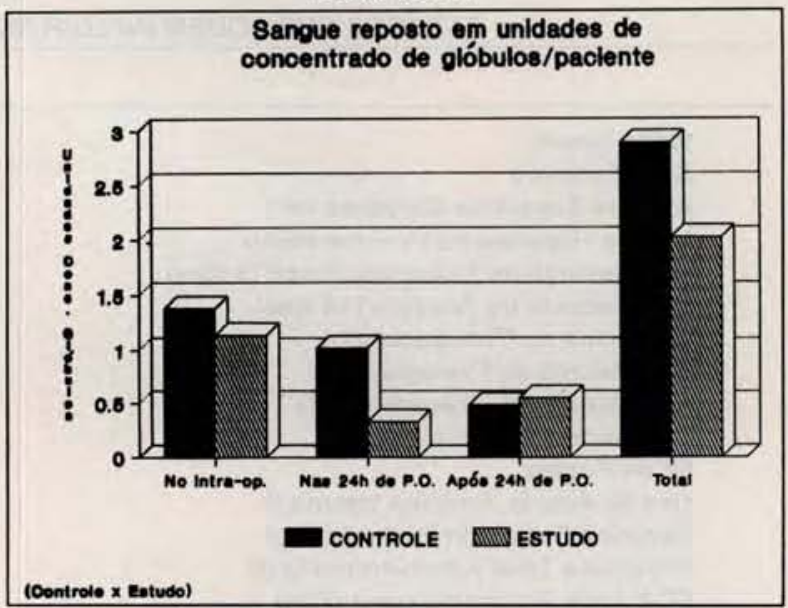

GRÁFICO 5

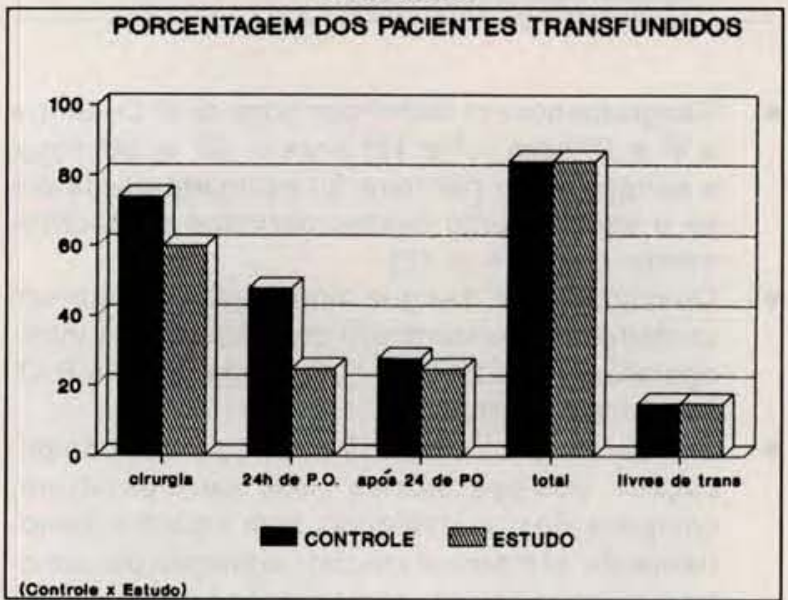

do sangramento da ordem de $56 \%$ em relaçāo ao grupo-controle (Gráfico 3 ). O sangramento por hora foi estatisticamente menor em todos os horários no grupo tratado.

As médias das unidades de sangue homólogo transfundido por paciente no grupo-controle, nos períodos referidos (intra-operatórios), $24 \mathrm{~h}$ de P.O., após $24 \mathrm{~h}$ de P.O. e total da internaçäo), foram 1,$379 ; 1,025 ; 0,487$ e 2,892 . As médias para o grupo-estudo foram 1,$141 ; 0,333 ; 0,549$ e 2,023 Diferença estatisticamente significante foi demonstrada nas 24 h de P.O. (Gráfico 4).

Com relação ao número de pacientes transfundidos nos respectivos horários, também houve diferença significante nas 24 horas de P.O. (48,7\% no grupo-controle e $25,4 \%$ no grupo-estudo), porém nāo houve diferença significante entre grupos com relaçāo ao número de pacientes que passaram o período de internaçăo sem receber sangue $(15,6 \%$ e 15,3\%) (Gráfico 5). 
VARGAS, G. F.; BRANCO, J. N. R.; GUIMARÄES, A. H. S.; KOBATA, C.; SILVA, E. T. V. F.; TELES, C. A.; LA ROTTA, C. A. A.; BATISTAFILHO, M. L. A.; ANDRADE, J. C. S.; BUFFOLO, E. - Ácido tranexâmico e hemostasia em cirurgia de revascularizaçāo do miocárdio com circulaçāo extracorpórea. Rev. Bras. Cir. Cardiovasc., 7(4):275-282, 1992.

TABELA 2

COMPLICAÇŌES

\begin{tabular}{|c|c|c|}
\hline & CONTROLE & ESTUDO \\
\hline $\begin{array}{l}\text { IAM Per-operatório } \\
\text { Complicaçōes Neurológicas }\end{array}$ & $7,5 \%$ & $7,2 \%$ \\
\hline $\begin{array}{l}\text { sem Seqüelas } \\
\text { Complicaçōes Neurológicas }\end{array}$ & $2,5 \%$ & $12,7 \%$ \\
\hline com Seqũelas & $5 \%$ & $3,6 \%$ \\
\hline Complicaçôes Renais & $5 \%$ & $10,9 \%$ \\
\hline Intubaçāo Prolongada & $5 \%$ & $7,2 \%$ \\
\hline Reoperação por Šangramento & $0 \%$ & (*) $1,8 \%$ ( 1 caso) \\
\hline
\end{tabular}

(") Achado cirúrgico:coágulo retido.

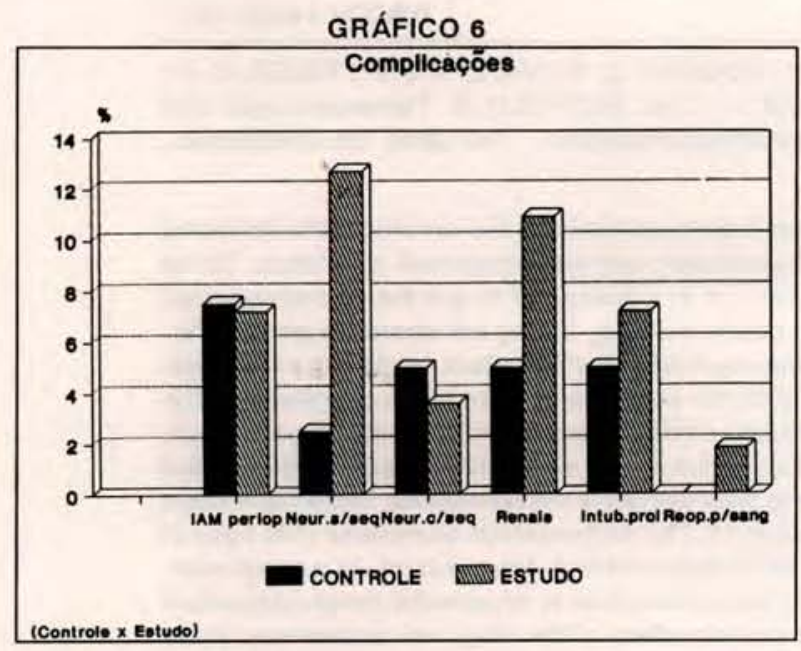

As complicaçōes pós-operatórias encontradas nos dois grupos encontram-se referidas na Tabela 2 e Gráfico 6.

Apesar de uma evidente maior tendência a complicaçōes no grupo-estudo, principalmente no que se refere a alteraçōes neurológicas e renais, a análise estatística não detectou diferença significante entre grupos.

\section{COMENTÁRIOS}

Sem dúvida alguma o ácido tranexâmico, na dose e modo administrado, possui efeito hemostático importante em CEC. Iniciamos a administração da droga já na fase da induçāo anestésica, visto que os ativadores teciduais do plasminogênio são liberados na fase da esternotomia ${ }^{29}$. O fato de usar a droga antes e durante a CEC também pode ser importante para o efeito hemostático da mesma, por uma possível ação protetora sobre as plaquetas ${ }^{27}$. 28, 46, o que nāo ocorreria se a droga fosse adminis- trada após a CEC. Tal impressionante efeito hemostático, no entanto, não se refletiu em uma igualmente impressionante diminuiçāo de transfusāo de sangue homólogo. Se considerarmos o período total de internaçāo, houve uma diminuiçāo no volume das transfusōes da ordem de $30 \%$, porém, a análise estatística nāo revelou diferença significante nessa reduçăo. A porcentagem de pacientes livres de transfusāo durante a internaçāo foi igual nos dois grupos. Talvez o efeito poupador de transfusōes homólogas da droga se mostrasse mais evidente se fossem usadas outras medidas para se evitar tais transfusōes descritas na literatura, tendo o ácido tranexâmico efeito aditivo. A conduta de se aceitar certo grau de anemia normolêmica no P.O., proposta por COSGROOVE et alii "talvez pudesse evidenciar melhor os efeitos poupadores de transfusōes homólogas da droga (em nosso Serviço indicamos transfusōes com Hb $<10 \mathrm{~g} / 100 \mathrm{ml}$ ou HTC < 30\%). Com relação à análise das complicações pós-operatórias nos dois grupos estudados, não obtivemos diferença estatisticamente significante. No entanto, destaca-se a ocorrência de um número incomum de alteraçōes neurológicas sem seqüelas (sonolência, agitação psicomotora e desorientação têmporo-espacial reversíveis), assim como alteraçōes pós-operatórias de creatinina no grupo-estudo (apesar de nenhum paciente ter sido submetido a diálise). Tais alteraçōes foram por nós questionadas quanto a estarem relacionadas à dosagem da droga ou, talvez, à idade média do grupo-estudo, que se revelou maior.

A ocorrência de efeitos colaterais do ácido tranexâmico em outras especialidades cirúrgicas é controversa na literatura, com autores referindo sua total inocuidade ${ }^{3,4,52}$ e outros alertando para as possiveis complicaçōes do bloqueio do sistema fibrinolítico $2,36,42,43,53$.

A experiência do uso do ácido tranexâmico em CEC não é grande na literatura ${ }^{26}$. Os dados obtidos, de um modo geral, neste trabalho, não nos autorizam a indicação do seu uso rotineiro em cirurgia cardiovascular com CEC, pelo menos considerando-se a dosagem utilizada neste estudo. Provavelmente seu uso seria muito útil em casos de maior risco de sangramento cirúrgico, como nas reoperaçōes, aneurismas de aorta ou cirurgias complexas, nas quais se espera tempo prolongado de CEC; seu uso rotineiro, no entanto, em operaçōes com CEC necessita ainda de maior número de estudos, no que concerne à dosagem ideal e ao modo de administraçāo, assim como observaçōes sobre seus possíveis efeitos colaterais.

\section{CONCLUSŌES}

Do uso do ácido tranexâmico na dosagem de 
VARGAS, G. F.; BRANCO, J. N. R.; GUIMARĀES, A. H. S.; KOBATA, C.; SILVA, E. T. V. F.; TELES, C. A.; LA ROTTA, C. A. A.; BATISTA FILHO, M. L. A.; ANDRADE, J. C. S.; BUFFOLO, E. - Ácido tranexâmico e hemostasia em cirurgia de revascularização do miocárdio com circulação extracorpórea. Rev. Bras. Cir. Cardiovasc., 7(4):275-282, 1992.

$10 \mathrm{~g}$ por via intravenosa, durante o ato operatório, em pacientes coronariopatas submetidos a cirurgia de revascularizaçāo do miocárdio com circulação extracorpórea, concluímos:

- Diminuiçāo de maneira significativa do sangramento no P.O.

- Diminuição significativa da quantidade de sangue homólogo transfundido por paciente, nas primeiras 24 horas de P.O.

- Tendência, apesar de nāo ter havido diferença estatisticamente significante, de maior ocorrência de complicaçōes neurológicas e renais no P.O.

- Necessidade de novos estudos, com dosagens menores e ampliaçāo da casuística para o estabelecimento de sua utilidade clínica no futuro.

\section{RBCCV 44205-190}

VARGAS, G. F.; BRANCO, J. N. R.; GUIMARÄES, A. H. S.; BOBATA, C.; SILVA, E. T. V. F.; TELES, C. A.; LA ROTTA, C. A. A.; BATISTA F ${ }^{\circ}$ M. L. A.; ANDRADE, J. C. S.; BUFFOLO, E. Tranexamic acid and hemostasis in myocardial revascularization with extracorporeal circulation. Rev. Bras. Cir. Cardiovasc., 7 (4):275-282, 1992

ABSTRACT: The synthetic antifibrinolytic drug tranexamic acid was evaluated in its hemostatic and blood saving effects, in patients submitted to myocardial revascularization with extracorporeal circulation. To 40 patients were administered placebo and to 55 tranexamic acid I.V. in a dosage of $10 \mathrm{~g}$ in the operative period ( $2 \mathrm{~g}$ in the anesthetic induction and the remaining $8 \mathrm{~g}$ in a continuous way during the operative procedure). Tranexamic acid, in this dosage, has proved to have a very impressive hemostatic effect, leadir 3 to a reduction in post operative bleeding of $47 \%$ in $12 \mathrm{~h}, 42,5 \%$ in $24 \mathrm{~h}$ and $40,5 \%$ when drains were taken off, related to the control group $(p<0.05)$. Tranexamic acid have led to less utilization for homologous paked red cells per patient, but statistical significance was found only in the $24 \mathrm{~h}$ of post operative period, with 1,025 units/patient in control group and 0,333 units/ patient in treated group. Concerning post operative complications, there have been more neurological alterations without sequelae $(2.5 \%$ against $12.7 \%)$ and creatinin alterations $(5 \%$ against $10.9 \%$ ) in the tranexamic acid group. Such alterations were attributed to the high dosage used. As a conclusion, we do not recommend routine use of tranexamic acid to patients submitted to myocardial revascularization in the dosage of $10 \mathrm{~g} \mathrm{I.V.,} \mathrm{but,} \mathrm{owing} \mathrm{to} \mathrm{the} \mathrm{evident} \mathrm{hemostatic} \mathrm{effect} \mathrm{of} \mathrm{the} \mathrm{drug,} \mathrm{we} \mathrm{recommend} \mathrm{more}$ investigations concerning the ideal dosage and way of administration.

DESCRIPTORS: tranexamic acid; surgical hemostasis; myocardial revascularization, surgery; extracorporeal circulation.

\section{REFERÊNCIAS BIBLIOGRÁFICAS}

ADDONIZIO Jr., V. P.; STRAUSSS III, J. F.; MACARAK, E. J.; COLMAN, R. W.; EDMUNDS Jr., L. H. Preservation of platelet number and function with prostaglandin E 1 during total cardiopulmoary bypass in rhesus monkeys. Surgery, 83: 619-625, 1978.

ASTEDT, B. \& LIEDHOLM, P. - Tranexamic acid and fibrinolytic activity of the vessel wall. Experientia, 30: $776-777,1974$.
4 BEKÁSSY, Z. \& ASTEDT, B. - Treatment with the fibrinolytic inhibitor tranexarr.ic acid: risk for thrombosis? Acta Obstet. Gynecol. Scand., 69: 353-354, 1990. (Carta ao Editor).

5 BICK, R. L. - Alterations of hemostasis associated with cardiopulmonary bypass:' pathophysiology, prevention, diagnosis and management. Semin. Thromb. Hemost., 3: 59-82, 1976.

6 BIRNBAUM, D. E. \& HOFFMEISTER, H. E. - Blood saving in open heart surgery. Stuttgart, Schattuer, $1990,111 \mathrm{p}$.

7 BLAUHUT, B.; GROSS, C.; NECEK, S.; DORAN, J. E.; SPATH, P.; LUNDSGAARD-HANSEN, P. - Effects of high-dose aprotinin on blood loss, platelet function, 
VARGAS, G. F.; BRANCO, J. N. R.; GUIMARÄES, A. H. S.; KOBATA, C.; SILVA, E. T. V. F.; TELES, C. A.; LA ROTTA, C. A. A.; BATISTA FILHO, M. L. A.; ANDRADE, J. C. S.; BUFFOLO, E. - Ácido tranexâmico e hemostasia em cirurgia de revascularizaçäo do miocárdio com circulação extracorpórea. Rev. Bras. Cir. Cardiovasc., 7(4):275-282, 1992.

fibrinolysis, complement, and renal function after cardiopulmonary bypass. J. Thorac. Cardiovasc. Surg., 101: 958-967, 1991.

BLOMBACK, M.; NORÉN, I.; SENNING, A. - Coagulation disturbances during extracorporeal circulation and postoperative period. Acta Chir. Scand., 127: 433 445, 1964.

CAN drugs reduce surgical blood loss? Lancet, 1:155156, 1988. (Editorial).

COCHRAN, W. G. - Some methods for strenghtering the Comman $\mathrm{X}^{2}$ test. Biometrics, 10: $417-451,1954$.

11 COSGROVE, D. M.; LOOP, F. D.; LYTLE, B. W.; GILL, C. C.; GOLDING, L. R.; TAYLOR, P. C.; FORSYTHE, S. B. - Determinants of blood utilization during myocardial revascularization. Ann. Thorac. Surg., 40: $380-384,1985$

DERMAN, U. M.; RAND, P. W.; BARKER, N. - Fibrinolysis after cardiopulmonary bypass and its relationship to fibrinogen. J. Thorac. Cardiovasc. Surg., 51: 223-225, 1966.

DIETRICH, W.; BARANKAY, A.; DILTHEY, G.; HENZE, R.; NIEKAU, E.; SEBENING, F.; RICHTER, J. A. Reduction of homologous blood requirement in cardiac surgery by intraoperative aprotinin application: clinical experience in 152 cardiac surgical patients. Thorac. Cardiovasc. Surg., 37: 92-98, 1989.

14 DIETRICH, W.; SPANNAGL, M.; KOCHUM, M.; WENDT, P.; SCHRAMM, W.; BARANKAY, A.; SEBENING, F.; RICHTER, J. A. - Influence of high-dose aprotin in treatment on blood loss and coagulation patterns in patients undergoing myocardial revascularization. Anesthesiology, 73: 1119-1126, 1990.

15 EDMUNDS Jr., L. H.; ELLISON, N.; COLMAN, R. W.; NIEWLAROWSKI, S.; RAO, A. K.; ADDONIZIO Jr., V. P.; STEPHENSON, L. W.; EDIE, R. N. - Platelet function during cardiac operation: comparison of membrane and bubble oxygenators. J. Thorac. Cardiovasc. Surg., 83: 805-812, 1982.

16 FISH, K. J.; SARQUIST, F. H.; VAN STEENNIS, C.; MITCHELL, R. S.; HILBERMAN, M.; JAMIESON, S. W.; LINET, O. I.; MILLER, D. C. - A prospective, randomized study of the effects os protacyclin on platelets and blood loss during coronary bypass operations. J. Thorac. Cardiovasc. Surg., 91: 436-442, 1986.

17 FRIEDENBERG, W. R.; MYERS, W.O.; PLOTKA, E. D.; BEATHARD, J. N.; KUMMER, D. J.; GATLIN, P. F.; STOIBER, D. L.; RAY III, J. F.; SAUTTER, R. D. Platelet dyisfunction associated with cardiopulmonary bypass. Ann. Thorac. Surg., 25: 298-305, 1978.

18 GANS, H.; SUBRAMANIAN, V.; JOHN, S.; CASTANNEDA, A. R.; LILLEHEI, C. W. - Theoretical and practical (clinical) considerations concerning proteolytic enzymes and their inhibitors with particular reference to changes in the plasminogen-plasmin system observed during assisted circulation in man. Ann. N. Y. Acad. Sci., 146: 721-736, 1968.

GOMES, M. M. R. \& McGOON, D. C. - Bleeding patterns after open-heart surgery. J. Thorac. Cardiovasc. Surg., 60: 87-97, 1970.

GOMES, O. M.; FREITAS NETO, A. G.; WOLOSKER, M.; LANGER, B.; DUBIEU, W. K.; ZERBINI, E. J. Antifibrinolíticos e circulaçāo extracorpórea. Rev. Hosp. Clin. Fac. Med. S. Paulo, 27: 209-213, 1972.

GRAM, J.; JANETZKO, T.; JESPERSEN, J.; BRUHN, H. D. - Enhanced effective fibrinolysis following the neutralization of heparin in open heart surgery increases the risk of post-surgical bleeding. Thromb. Haemost., 63: 241-145, 1990.

HAVEL, M.; TEUFELSBAUER, H.; KNOBL, P.; DALMATINER, R.; JAKSCH, P.; ZWOLFER, W.; MULLER, M.; VUKOVICH, T. - Effect of intraoperative aprotinin administration on postoperative bleeding in patients undergoing cardiopulmonary bypass operation. $J$. Thorac. Cardiovasc. Surg., 101: 968-972, 1991.

HARDER, M. P.; EIJSMAN, L.; ROOZENDALL, K. J.; VAN OEVEREN, M.; WILDEVUUR, C. R. H. Aprotinin reduces intraoperative an postoperative blood loss in membrane oxygenator cardiopulmonary bypass. Ann. Thorac. Surg., 51: 936-941, 1991.

4 HOLLANDER, M. \& WOLFE, D. A. - Nonparametric statistical methods. New York, John Wiley \& Sons, 1973. 503p.

HOPE, A. F.; HEYNS, A.; LOTTER, M. G.; VAN REENEN, O. R.; KOCK, F.; BADENHORST, P. N.; PIETERS, H.; KOTZE, H.; MEYER, J. M.; MINNAAR, P. C. - Kinetics and sites of sequestration of indium 111 - labeled human platelets during cardiopulmonary bypass. J. Thorac. Cardiovasc. Surg., 81: 880-886, 1981.

HORROW, J. C.; HLAVACEK, J.; STRONG, M. D.; COLLIER, W.; BRODSKY, I.; GOLDMAN, S. M.; GOEL, I. P. - Prophylactic tranexamic acid decreases bleeding after cardiac operations. J. Thorac. Cardiovasc.Surg., 99: 70-74, 1990.

HORROW, J. C.; VAN RIPER, D. F.; STRONG, M. D.; BRODSKY, I.; PARMET, J. L. - Hemostatic effects of tranexamic acid and desmopressin during cardiac surgery. Circulation, 84: 2063-2070, 1991.

JONG, J. C. F.; NELEMS, J. M.; SIBINGA, C. T. S.; WILDEVUUR, C. R. H. - The influence of tranexamic acid on thrombocytopenia caused by artificial surfaces. Trans. Am. Soc. Artif. Intern. Organs, 208: 596-603, 1974

29 KEVY, S. V.; BERHARD, W. F.; McMAHON, C. I, Pathogenesis and prophylactic treatment of fibrinolysis in open-heart surgery. Surg. Forum., 16: 6566, 1965. 
VARGAS, G. F.; BRANCO, J. N. R.; GUIMARÄES, A. H. S.; KOBATA, C.; SILVA, E. T. V. F.; TELES, C. A.; LA ROTTA, C. A. A.; BATISTA FILHO, M. L. A.; ANDRADE, J. C. S.; BUFFOLO, E. - Ácido tranexâmico e hemostasia em cirurgia de revascularizaçāo do miocárdio com circulaçāo extracorpórea. Rev. Bras. Cir. Cardiovasc., 7(4):275-282, 1992.

KIRKLIN, J. W. \& BARRAT-BOYES, B. G. - Hipothermia, circulatory arrest and cardiopulmonary bypass. In: Cardiac surgery. New York, John Wiley \& Sons, 1986. p. 29-82.

31 KIRKLIN, J. W.; KIRKLIN, J. K.; LELL, W. A. Cardiopulmonary bypass for cardiac surgery. In: SABISTON Jr., D. C. \& SPENCER, F. C. - Gibbon's surgery of the chest. 4. ed. Philadelphia, W. B. Saunders Company, 1983. p. 909-925.

KUCUK, O.; KWAAN, H. C.; FREDERICKSON, J.; WADE, L.; GREEN, D. - Increased fibrinolytic activity in patients undergoing cardiopulmonary bypass operation. Am. J. Hematol., 23: 223-229, 1986.

LAMBERT, C. J.; MORENGO-ROWE, A. J.; LEVESON, J. E.; GREEN, R. H.; THIELE, J. P.; GEISLER, G. F.; ADAM, M.; MITCHEL, B. F. - The treatment os postperfusion bleeding using epsilon-aminocaproic acid, cryoprecipitate, fresh-frozen plasma, and protamine sulfate. Ann. Thorac. Surg., 28: 440-444, 1979.

34 LOURES, D. R. R.; VERGINELLI, G.; MARCIAL, M. B.; AMARAL, R. G.; ZERBINI, E. J. - Estudo da coagulação nos pacientes operados com circulaçāo extracorpórea sob o uso per-operatório de ácido trans-4amino-metil-ciclohexano carboxílico. Rev. Bras. Clin. Terap., 2: 161-166, 1973.

MAMMEN, E. F.; THAL, A. P.; KATZ, W. - Aplicaçāo do Trasylol na circulação extracorpórea. Rev. Bras. Med., 25: 483-487, 1986.

NAEYE, R. L. - Thrombotic state after a hemorragic diathesis, a possible complication of therapy with epsilon-aminocaproic acid. Blood, 19: 694-701, 1962.

OEVEREN VAN, W.; JANSEN, N. J. G.; BIDSTRUP, B. P.; ROYSTON, D.; WESTABY, S.; NEUHOF, H.; WILDEVUUR, C. R. H. - Effects of aprotinin on hemostatic mechanisms during cardiopulmonary bypass. Ann. Thorac. Surg., 44: 640-645, 1987.

OKAMOTO, S. \& OKAMOTO, U. - Amino-methylciclohexane-carboxylic acid (AMCHA): a new potent inhibitor of fibrinolysis. Keio J. Med., 11: 106-111, 1962.

OKAMOTO, S.; OSHIBA, S.; MIHARA, H.; OKAMOTO, $U$. - Synthetic inhibitors of fibrinolysis: in vitro and in vivo mode of action. Ann. N. Y. Acad. Sci., 146: 414-429, 1968.

OKAMOTO, S.; SATO, S.; TAKADA, Y.; OKAMOTO, U. - An active stereo-isomer (trans-form) of AMCHA and its antifibrinolytic (antiplasminic) action in vitro and in vivo. Keio J. Med., 13: 177-185, 1964.

41 PORTER, J. M.; SILVER, D.; SABISTON Jr., D. C. Alterations in fibrinolysis and coagulation associated

with cardiopulmonary bypass. J. Thorac. Cardiovasc. Surg., 56: 869-877, 1968.

42
RATNOFF, O. D. - Epsilon-aminocaproic acid: a dangerous weapon. N. Engl. J. Med., 280: 1124-1125, 1969.

RYDIN, E. \& LUNDBERG, P. O. - Tranexamic acid and intracranial thrombosis, Lancet, 2 (7975): 49, 1976 (Carta ao Editor).

SALZMAN, E. W.; WEINSTEIN, M. J.; WEITRANUB, R M.; WARE, J. A.; THURER, R. L.; ROBERTSON, L.; DONOVAN, A.; GAFFNEY, T.; BERTELE, V.; TROLL, J.; SMITH, M.; CHUTE, L. E.- Treatment with desmopressin acetate to reduce blood loss after cardiac surgery: a double trial. N. Eng. J. Med., 314: $1402-1406,1986$.

5 SIEGEL, S. (ed.) - Estatística nāo paramétrica para as ciências do comportamento, São Paulo, McGrawHill do Brasil, 1975.

6 SOSLAU, G.; HORROW, J. C.; BRODSKY, I. - Effect of tranexamic acid on platelet ADP during extracorporeal circulation. Am. J. Hematol., 38: 113-119, 1991.

47 TAMARI, Y.; ALEDORT, L.; PUSZKIN, E.; DEGRAN, T. J.; WAGNER, N.; KAPLITT, M. J.; PEIRCE II, E. C. - Functional changes in platelets during extracorporeal circulation. Ann. Thorac. Surg., 19:639-647, 1975.

48 TICE, D. A.; REED, G. E.; CLAUSS, R. H.; WORTH, M. $H$. - Hemorrhage due to fibrinolysis with open-heart operations. J. Thorac. cardiovasc. Surg., 46: 673$679,1963$.

9 TICE, D. A. \& WORTH, M. H. - Recognition and treatment of postoperative bleeding associated with open-heart surgery. Ann. N. Y. Acad. Sci., 146: 745-753, 1968.

TICE, D. A.; WORTH, M. H.; CLAUSS, R. H.; REED, G. H. - The inhibition of trasylol of fibrinolytic activity associated with cardiovascular operations. Surg. Gynec. Obstet., 119: 71-74, 1964.

51 VANDER SALM, T. J.; ANSEL, J. E.; OKIKE, O. N.; MARSICANO, T. H.; LEW, R.; STEPHENSON, W. P.; ROONEY, K. - The role of epsilon-aminocaproic acid in reducing bleeding after cardiac operation: a double-blind randomized study. J. Thorac. Cardiovasc. Surg., 95: 538-540, 1988.

52 VESTRAETE, M. - Clinical application of inhibitors of fibrinolysis. Drugs, 29: 236-261, 1985.

WOO, K. S.; TRE, L. K. K.; WOO, J. L. F.; OWEN, J. V. - Massive pulmonary thromboembolism after antifibrinolytic therapy. Ann. Emerg. Med., 18: 116117, 1989. 